(REVIEW ARTICLE)

\title{
The importance of nanotechnology and drug carrier systems in pharmacology
}

\author{
Yerebasan Ulku ${ }^{1}$ and Gul Baykalir Burcu ${ }^{2, *}$ \\ ${ }^{1}$ Department of Pharmacology and Toxicology, Faculty of Veterinary Medicine, Firat University, 23119 Elazig, Turkey. \\ 2 Department of Nursing, Faculty of Health Sciences, Firat University, 23119 Elazig, Turkey.
}

Publication history: Received on 21 January 2020; revised on 29 January 2020; accepted on 03 February 2020

Article DOI: https://doi.org/10.30574/gscbps.2020.10.2.0018

\begin{abstract}
Nanotechnology is one of the newest and most interesting areas of recent years. It offers innovations in diagnosis and treatment with the integration of limited size nanoparticles into larger living systems. It was found application in the field of microbiology, vaccine production, wound healing, tissue regeneration, and genetic, especially in pharmacology. It was significantly increased in the life quality of patients compared with traditional methods due to a number of superiorities such as demonstrated tumor specificity especially in the field of oncology, pass the blood brain barrier in neurodegenerative diseases and offer non-invasive alternatives in diabetes. However, there are also some risks of systemic, genetic and cytotoxic. With this study, it was aimed to inform about the status of nanotechnology in the field of pharmacology, advantages and drug carrier systems.
\end{abstract}

Keywords: Nanotechnology; Pharmacology; Carrier system; Targeting

\section{Introduction}

Nanotechnology, having a wide range of applications, is defined in various ways in the science world. The term of nano signifies one per one billion of any expression. Nanometer means its version that is adapted to the length. For example, a nanometer is a very short length corresponding to a billion for a meter, a million of a millimeter or a thousand of a micrometer. Also, the word "nano" is derived from the word "nanus" in Latin and "nanos" in Greek and it means "dwarf" [1]. Nanotechnology is a technology science related to design, formation, and usage of materials with nano-sized components and dimensions of these components range from $1 \mathrm{~nm}$ to $100 \mathrm{~nm}$ [2]. Nanotechnology is the processes carried out by controlling the matter at the molecular level. Medicine, devices and systems that are formed and used due to their small dimensions have different properties/functions.

It is possible to use nanotechnology in various fields such as medicine, pharmacy, food industry, veterinary medicine, and textile. Nanotechnology is thought to be originally designed for vaccines and cancer drugs [3]. Also, positive results have been obtained in the treatment of brain tumors since the drugs can be transported to the target site via blood and brain barrier [4].

All the nanosystems used provide some advantages when compared to conventional systems. If these advantages are summarized increasing the therapeutic effect by increasing the half-life of the drugs [5-7], reducing the side effects of the drugs [8], monitoring the release and distribution of the drugs, staying in circulation for a long term [4], monitoring of the treatment efficiency,developing effects without harming other systems and organs [9, 10], avoiding some drug use problems (drug application at night, forgetting to take/administer drugs, using drugs in short time intervals, etc.).

\footnotetext{
${ }^{*}$ Corresponding author

E-mail address: brcgul@firat.edu.tr
} 
As in any drug, nanotechnological product also has some disadvantages in addition to its advantages. These are high cost, failure to prepare all active substance formulations [11], possibility of causing immediate drug release/failure to withdraw the release at a desired time period [8], non-conformance with the biological system, nanoparticles may cause inflammation in the lung tissue because they enter the body from the lungs at the fastest [12], their small size and large surface area may cause particle aggregation, exceeding the desired volume of distribution and causing toxic effects by degrading the protein structure [13].

Physicochemical properties of nanomedicine such as size, surface load and hydrophilicity affect mucosal absorption $[14,15]$. It provides advantages in kinetics such as specific drug distribution, high metabolic stability, high membrane permeability, maximum bioavailability, and long-term drug release. Due to the increase in drug permeability, tight junctions between the endothelial cells are opened by means of nanomolecules and drugs are released into the cells. The transition especially realizes via the inhibition of P-glycoprotein systems [16]. Larger particles are rapidly removed from the bloodstream via the macrophages of the reticuloendothelial system (RES). Nanomedicine needs to be coated with surfactants in order to prolong their presence in circulation and to make phagocytes sensitive against foreign antigens. Essential surfactants are polyethylene glycol (PEG), polyethylene oxide, poloxamer, poloxamine, and polysorbate 80 [17-19].

\subsection{Drug Delivery Systems in Nanotechnology}

Nanotechnology focuses on the formulation of therapeutic agents within nanocarriers. These formulations provide the delivery of the targeted drugs to the diseased structure [20]. In recent years, significant developments have been made in the delivery systems of nanomedicine. Since nanotechnology applications in health field have become widespread and new drugs have taken the place of traditional drugs, the process has increasingly accelerated. Thanks to the advancement of the techniques that selectively deliver the drugs to pathological cells, tissues or organs, drug delivery systems have become the most important research area [21]. In other words, selective delivery of the active substance to the desired site occurs via the drug delivery systems.

Most frequently used carrier systems are nanoparticles, dendrimers, liposomes, polymer conjugates, and micelles. It can be considered that the number of drug delivery systems may further increase in the future as they have the potential to be used in the diagnosis and treatment of many diseases.

In the development of drug delivery applications, nanocarriers are designed in different pharmaceutical forms including oral, parenteral, pulmonary, nasal, transdermal, or topical formulations [22]. Delivery of the drug to the structure where it will activate is one of the main problems of pharmaceutical technology. Drug use is limited due to the situations such as low drug solubility, overdose and formation of toxicity, non-specific transfer, short half-life period etc. Today, many researchers participate in activities of developing new drug delivery systems in order to minimize the increasing problems of the drugs and to turn new developments into clinics [23]. However, the use of nanocarriers in drug release brings a number of problems such as not being suitable for low-potential drug releases, being hard to obtain and store them, and having possibility to cause genetic damages and mutations [20].

\subsubsection{Nanoparticles}

Nanoparticles are present in the forms of needles, spheres, prisms, and cubic bars. They are colloidal structures with dimensions varying between 10 and $100 \mathrm{~nm}$. They are formed of natural or synthetic macro molecules [24]. These are the systems where the active substance is dissolved, absorbed or bound in the particle. In the pharmaceutical sector, their practical use in the applications such as direct binding to the active substance, confinement, and targeting has brought them into a preferred position [20]. They are called as nanocapsules or nanospheres according to their preparation techniques [25]. Nanocapsules are vesicular structures. The drug is confined in a cavity and surrounded by a polymer membrane. Nanospheres are the matrix systems in which the drug is physically dispersed.

Nanoparticles provide advantages due to some of its characteristics. One of them is that they have small particle sizes. Thus, they pass through the small capillary and are taken into the cell, and it is provided to accumulate the active substance in the target site. Other one is the use of biosoluble materials in the preparation stage of nanoparticles. By means of these biosoluble materials, controlled release of active substance is provided in the target tissue in periods lasting for days and/or weeks.

\subsubsection{Liposomes}

These are biocompatible, reversible vesicular structures having double phospholipid layers and sizes ranging from nanometers to a few micrometers [23]. They are widely used as a pharmaceutical carrier due to its biopharmaceutical 
characteristics. They have some advantages such as high encapsulation efficiency for both hydrophilic and hydrophobic therapeutic substances, providing the encapsulated drugs protected from physical factors, targeting specific cells, tissues and related organs, providing long-term blood circulation with the use of inert/biocompatible polymers, and realizing a controllable size [26-29].

Various problems such as low solubility, short half-life period, low bioavailability, and strong side effects of the drugs can be widely solved by liposomes. In general, liposomes are used to reduce the toxicity and side effects caused by the drugs. In recent years, numerous liposome formulations are approved. First FDA-approved drug in liposomal formulation is Doxil [30].

\subsubsection{Dendrimers}

Dendrimers are the molecules having a core at molecular center and recurrent branches around the core [31, 32]. Due to its high encapsulation capability and high water solubility, they are considered as ideal delivery systems [33]. In order to their feature of high functionality, they enable high drug delivery capacity on the surface [34]. In recent times, they have been used in various cancer treatments [35-38].

\subsubsection{Micelles}

They are spherical structures having a particle size ranging from $5 \mathrm{~nm}$ to $100 \mathrm{~nm}$ and being composed of core made of hydrophobic blocks a robust shell with hydrophilic polymer chain. The core is formed from hydrophobic polymers such as polycaprolactone (PCL), polypropylene glycol (PPG) or poly D,L-lactide (PAL), and the shell is made of hydrophilic PEG [34]. Phospholipid micelles are less toxic and they enable a longer blood circulation [39]. If the micelles are PEG, they become more functional. The examples of this group of drugs can be specified as NK012 and NK105 that are used in cancer treatment [40]. Micelles increase the bioavailability of the drugs with low-solubility by enabling them to be soluble, and they can remain in the body for a sufficiently long time in order to allow the active substance to be accumulated in the target tissue [23].

\subsubsection{Polymer conjugates}

When peptide-protein substances are taken orally, they are usually degraded in the stomach and their half life is very short in parenteral administration. This causes problems in the delivery of the drug in the body and in its pharmacokinetics. Thus, polymers are highlighted in order to eliminate such problems. Most commonly used polymers for this purpose include PCL, polyethylene (PE), PEG, polyethylene oxide (PEO), polylactic acid (PLA), polylacticoglycolic acid (PLGA), and polyglycolic acid (PGA) [25]. Today, the most frequently used polymer in the formulation of peptide-protein drugs is PEG.

\subsubsection{Nanogels}

Nanogels are structures having sizes ranging from $20 \mathrm{~nm}$ to $200 \mathrm{~nm}$ that are in the liquid state at room temperature and in the gel state at body temperature. They do not dissolve in water and biological liquids. Since their most important characteristic is swelling, nanogels can bind drugs and biological substances. It also improves the solubility and stability of the low soluble drugs $[41,42]$. Additionally, it is a new drug carrier increasing the cellular uptake potentials more than a free drug. It is preferred due to its inert structure in the blood circulation (does not produce any immunological response), high biocompatibility and biodegradability (in order to prevent the deposition in the body), and facilitating the crossing of the blood brain barrier [43].

\subsubsection{Nanocrystals}

They are defined as nano-sized crystals or the active substances in the crystal structure. They are developed to increase the bioavailability of the drugs with low solubility. In addition, due to its high bioavailability, it has several advantages such as being able to be used at lower doses, being able to prepare drugs with water solubility problems, and being able to be administered easily $[44,45]$.

\subsubsection{Solid lipid nanoparticles}

This group consists of surfactants and water. Solid lipids, triglycerides, PEG-coated lipids, wax, and steroids are used in its structure [41]. They have advantages such as being added to lipophilic and hydrophilic active substances, protecting the encapsulated active substances from external factors, and controlling the release of active substances [44]. 


\subsubsection{Niosomes}

They are the structures formed by non-ionic surfactants in an aqueous environment [45]. Since hydrophilic, hydrophobic and amphiphilic structures take place in the structure of niosomes, they provide a broad spectrum for the solubility of the active substance of the drug [41] and they are used as a carrier for the substances with low solubility [46].

\subsubsection{Nanoemulsions}

They are heterogeneous structures in which two immiscible liquids are dispersed in the form of droplets. Their size varies between 20 and $200 \mathrm{~nm}$ [47]. They are formed of hydrophilic and lipophilic phases. At least three components are required to conduct the formulation of an emulsion: Fat phase, water phase and surfactant [48]. They increase the absorption of different active substances via oral, nasal, parenteral, transdermal or ocular ways.

\subsection{Drug Targeting Methods in Nanotechnology}

Targeting systems have been developed to minimize the degradation or loss of the active substance, to reduce the amount of drug required for a therapeutic effect, to increase bioavailability, to extend the half-life of the drug, to prohibit the strong adverse effects, and to reduce the treatment costs [23]. The targeted drugs are beneficial for cancer, immune system diseases, cardiovascular system diseases, and central nervous system diseases.

\subsubsection{Passive targeting}

Passive targeting is the transferring of the particles to specific sites via passive factors or natural physiological processes [41]. Anatomical differences between normal and pathological tissues are benefited. When the tumors show loose vascularization, it spontaneously permeates in the interstitium via the drug delivery system [49]. This characteristic is defined as the EPR (increased permeability and retention) effect enabling the deposition of nanocarriers. It provides the nanocarriers to be kept at the tumor site for a long time [50].

There are some factors affecting passive targeting. Particle size is a factor determining the drug stability and drug release. Particle size should be small in order for nanocarriers not to be caught by RES. However, the diameter should be at least $10 \mathrm{~nm}$ in order to prevent vacuolation $[51,52]$. An ideal nanocarrier size should have a diameter between 10 and $150 \mathrm{~nm}$ [53]. As the surface area-volume ratio of the small particles is larger, the drug release is faster because the drug molecule is closer to the surface. Large particles involve more molecules and release more slowly [54]. Another factor is surface. It is a factor that determines the hydrophobicity of nanoparticles. For a successful transport, the period of the nanoparticle circulation should be increased. This is possible by coating them with hydrophobic polymers (PEG, polysorbate 80, poloxamine, PEO) [55]. In addition, active substance loading capacity and release rate affect passive targeting.

\subsubsection{Active targeting}

Active targeting occurs by using vectors having high site-specific affinity. They are the interactions between the carrier system and the target cell or tissue [56]. Targeting ligands expressed in the target site are added to the nanocarrier surface. Ligand is selected in such a way to bind to a receptor highly expressed by tumor cells and not expressed by normal cells. Active targeting of nanocarriers can be achieved by adding the targeting ligands such as monoclonal antibodies, transferrin, various peptides, folate, aptamers, hyaluronic acid, biotin, albumin (single chain oligonucleotides), or certain sugars on their surface [57]. Via active targeting, the side effects of nanomedicine are reduced by increasing their cytotoxic effect and the development of multi-drug resistance is prevented [58].

\subsubsection{Direct application on the tissue and organ}

The drug is directly applied on the target tissue or organ. For example, in myocardial infarction, it is the direct administration of thrombolytic enzymes into the coronary vessels [59].

\subsubsection{Physical targeting}

The purpose is the temperature and $\mathrm{pH}$-based targeting in different tissues and organs. Nanoparticles that perform $\mathrm{pH}$ based release accumulate more in these tissues and they release more [60]. 


\subsection{Usage Areas of Nanotechnology}

\subsubsection{Cancer treatment}

The whole body is affected during the treatment due to the non-specific chemotherapy agents used, today. As the current chemotherapy is aimed at destroying all the rapidly dividing cells, it can also damage the rapidly proliferating cells such as hair follicles and intestinal epithelium [61]. Cancer treatment with nanoparticles is presented as a new option [62]. In the treatment performed with nanoparticles, other tissues and organs other than the diseased area are not affected [9, 10]. Additional, micelle and liposome have become alternative to chemotherapy since drug concentration is increased by adding PEG. Genexol-PM (paclitaxel) that is used in the treatment of breast cancer is clinically approved [40]. Dendrimers are multi-branch molecules having various functional groups for targeting, imaging etc. [63]. In vitro imaging, localization (folic acid) and targeting of methotrexate that is an anti-cancer drug are the developments associated with the dendrimer system [64]. Another promising nanoparticle form in cancer treatment is the carbon nanotubes [3]. The water-insoluble drugs can be easily loaded accordingly [65]. Also, it is reported that nanocrystals increase the cytotoxicity of chemotherapeutic agents [66].

\subsubsection{Wound healing}

In recent years, nanoparticles are topically used in wound healing. Gold nanoparticles are used as hydroxypropyl methyl cellulose (HPMC) gels in order to reduce the side effects of systemic treatment [67]. The slower release of the nanoparticles than the gel increases the retention time on the skin [68]. Studies are planned to use electrospin nanofibers in diabetic ulcers, burns, and various skin infections.

\subsubsection{Treatment of infectious diseases}

Throughout the world, viral infections affect the health of millions of people and constitute major global health problems. They cause negative effects both on health and socioeconomic development [69]. Abacavir et al., conducted an in vitro examination on Anti-Hiv activity of lamivudine [70]. T-cells take place among the important components of the immune system [71]. Thus, suppression of T-cells play an important role in the formation of infections [72]. Jaramillo-Ruiz et al. [73], showed for the first time that carboxylan dendrimers can be used in the prevention of infections developing as a result of HIV and the suppression of T-cells.

New researches have been conducted for Hepatitis B virus (HBV) by using nanotechnology. In their study Wang et al. [74] showed that metoxypolyethylene glycol having a polyethyleneimine (PEI) layer showed the highest Anti-HBV effect. Chitosan, which is non-toxic, can be biologically degraded, and has a good biological profile, is used against HPV [75]. In addition, in the study by Moon et al. [76], mice that did not respond to the treatments with traditional drugs showed strong Anti-HCV (Hepatitis C virus) activity in the treatment performed with nanoparticles. It is reported that Vivagel that is used against the Zika virus (ZIKV) has a strong antiviral activity against ZIKV [33].

There are some points that should be considered in the use of parenteral vaccines. These points are eliminated by the use of nanoparticles (trained medical personnel, danger of reutilization of the needles, high dose regimens, immune reactions, etc.) [77].

\subsubsection{Diabetes treatment}

Since the current treatments for the diabetic patients are painful, invasive, and requires continuous dose adjustment, nanotechnological applications have been sought. Oral insulin use is one of the most suitable methods. However, reductions occur in its effect by being affected by the stomach enzymes. Insulin should be protected against degradation while passing through the gastrointestinal system. In animal studies, insulin polymer nanoparticles are used orally in the form of pellet feeds. N-isopropylacylamide, PEI, and polymetacrylic acid are some of these polymers.

Chitosan provides the nanoparticles to be used in the form of inhalation. Chitosan with a mucoadhesive feature can transfer the nanoparticular drug to the blood circulation without degradation [78-81]. Another method is 'Smart Tattoo'. Decreased glucose level in interstitial fluid is determined through polyethylene nanosensors coated with fluorescent molecules subcutaneously placed in order to monitor glucose levels [82].

Also, artificial pancreas is considered as a permanent method for diabetic patients. Silicone box with a diameter of 20 $\mathrm{nm}$ is coated with nanopores [83]. It is aimed to replace the healthy beta cells taken from the animals with nonfunctional cells. Increase in the size of nanopores may cause immune reactions [84]. In addition, it is reported that biokil socks effectively reduce bacterial growth in diabetic patients [85]. 


\subsubsection{Atherosclerosis treatment}

Nanotechnological drugs provide treatment alternatives for cardiovascular diseases at a cellular level. Chemoreceptors placed in the carbon nanotechnological device can differentiate the plaques in the arterial wall. This device can clean the plaques on the arterial wall by vacuuming [86]. Also, nanostents are developed to provide the continuity of the circulatory system. They contribute to the healing process of cardiac patients [87].

\subsubsection{Osteoporosis treatment}

Osteoporosis has the highest prevalence among the bone diseases. Various drugs have been studied for osteoporosis treatment. Among these drugs, Risedronat increases the bioactivity of osteoblasts and the bone regeneration [88]. In another study [89], bone regeneration is provided by administering magnetic nanoparticles directly to the bone for osteoporosis treatment.

In another study [90], it is reported that nanocalcium carbonate and nanocalcium citrate applications have increased the bone mineral density in the rats undergoing ovariectomy. In the study by Tokudome et al. [91], tricalcium phosphate nanoparticles containing zinc were injected to the rats with osteoporosis and positive results are obtained as a result of the jawbone mineral density measurements.

\section{Conclusion}

The present study includes current pharmacologic data regarding nanotechnology and drug delivery systems. Today, concern regarding the nanomedicine increases every passing day due to the insufficient treatments via conventional methods and the excessive side effects of the drugs used. However, even though the drugs produced by nanotechnology offer innovations/conveniences in the diagnosis and treatment of various diseases, their high costs and their longunknown history inhibit them to be used commonly. It is considered that a revolution can be done in health sector by eliminating these two factors. The reason is that it is estimated that they will significantly improve the qualities of life of the patients in the fighting against diseases. The use of nanotechnology will contribute to the development of new particles and carrier systems in the future. It will be inevitable that nanomedicine will bring great innovations to the health sector in the forthcoming years. With increasing of the studies in the future, it is thought that nanotechnology would take a broader place in diagnosis and treatment of diseases leading to death.

\section{Compliance with ethical standards}

\section{Disclosure of conflict of interest}

There is no conflict of interest.

\section{References}

[1] Chakravarthi VP and Balaji N. (2010). Applications of nanotechnology in veterinary medicine. Veterinary World, $25,477-480$.

[2] Tatli Seven P, Seven I, Gul Baykalir B, Iflazoglu Mutlu S and Salem AZM. (2018) Nanotechnology and nanopropolis in animal production and Health: an overview. Italian Journal of Animal Science, 1-10.

[3] Sanginario A, Miccoli B and Demarchi D. (2017). Carbon nanotubes as an effective opportunity for cancer diagnosis and treatment. Biosensors, 7(1), 1-23.

[4] Mohanraj VJ and Chen Y. (2006). Nanoparticles - a review. Tropical Journal of Pharmaceutical Research, 5(1), 561-573.

[5] Agrawal M, Saraf S, Saraf S, Antimisiaris SG, Chougule MB, Shoyele SA and Alexander A. (2018). Nose-to-brain drug delivery: an update on clinical challenges and progress towards approval of anti-Alzheimer drugs. Journal of Controlled Release, 281, 139-177.

[6] Srimathi U, Nagarajan V and Chandiramouli R. (2018). Interaction of Imuran, Pentasa and Hyoscyamine drugs and solvent effects on graphdiyne nanotube as a drug. Journal of Molecular Liquids, 265, 199-207.

[7] Wang L, Yao J, Zhang X, Zhang Y, Xu C, Lee RJ, Yu G, Yu B and Teng L. (2018). Delivery of paclitaxel using nanoparticles composed of poly (ethylene oxide)-b-poly(butylene oxide) (PEO-PBO). Colloids Surf B Biointerfaces, 161, 464-470. 
[8] Wilczewska AZ, Niemirowicz K, Markiewicz KH and Car H. (2012). Nanoparticles as drug delivery systems. Pharmacological Reports, 64(5), 1020-1037.

[9] Huang B, Abraham WD, Zheng Y, Bustamante Lopez SC, Luo SS and Irvine DJ. (2015). Active targeting of chemotherapy to disseminated tumors using nanoparticle-carrying $\mathrm{T}$ cells. Science Translational Medicine, 7(291), 1-21.

[10] Shen B, Ma Y, Yu S and Ji C. (2016). Smart multifunctional magnetic nanoparticle-based drug delivery system for cancer thermo-chemotherapy and intracellular imaging. ACS Applied Materials and Interfaces, 8, 24502-24508.

[11] Ravi Kumar MN. (2000). Nano and microparticles as controlled drug delivery devices. Journal of Pharmacy and Pharmaceutical Sciences, 3(2), 234-258.

[12] Duffin R, Mills NL and Donaldson K. (2007). Nanoparticles-a thoracic toxicology perspective. Yonsei Medical Journal, 48(4), 561-572.

[13] Syed S, Zubair A and Frieri M. (2013). Immune response to nanomaterials: implications for medicine and literature review. Current Allergy and Asthma Reports, 13(1), 50-57.

[14] Petros RA and DeSimone JM. (2010). Strategies in the design of nanoparticles for therapeutic applications. Nature Reviews Drug Discovery, 9(8), 615-627.

[15] Roger E, Lagarce F, Garcion E and Benoit JP. (2010). Biopharmaceutical parameters to consider in order to alter the fate of nanocarriers after oral delivery. Nanomedicine (Lond), 5(2), 287-306.

[16] Modi G, Pillay V, Choonara YE, Ndesendo VM, du Toit LC and Naidoo D. (2009). Nanotechnological applications for the treatment of neurodegenerative disorders. Progress in Neurobiology, 88, 272-285.

[17] Jain A and Jain SK. (2008). In vitro and cell uptake studies for targeting of ligand anchored nanoparticles for colon tumors. European Journal of Pharmaceutical Sciences, 35(5), 404-416.

[18] Francis MF, Cristea M and Winnik FM. (2005). Exploiting the vitamin B12 pathway to enhance oral drug delivery via polymeric micelles. Biomacromolecules, 6(5), 2462-2467.

[19] Mori S, Matsuura A, Rama Prasad YV and Takada K. (2004). Studies on the intestinal absorption of low molecular weight heparin using saturated fatty acids and their derivatives as an absorption enhancer in rats. Biological and Pharmaceutical Bulletin, 27(3), 418-421.

[20] Tuylek Z. (2017). Drug delivery systems and nanotechnological interaction. Bozok Medical Journal, 7(3), 89-98.

[21] Vasir JK and Labhasetwar V. (2005). Targeted drug delivery in cancer therapy. Technology in Cancer Research and Treatment, 4(4), 363-374.

[22] Onoue S, Sato H, Ogawa K, Kojo Y, Aoki Y, Wada K, Mizumoto T and Yamada S. (2012). Inhalable dry-emulsion formulation of cyclosporine A with improved anti-inflammatory effects in experimental asthma/COPD-model rats. European Journal of Pharmaceutics and Biopharmaceutics, 80(1), 54-60.

[23] Celik M. (2014). Drug targeting techniques in pharmaceutical biotechnology. Ph.D. thesis, University of Erciyes, Kayseri.

[24] Cushing BL, Kolesnichenko VL and O'Connor CJ. (2004). Recent advances in the liquid-phase syntheses of inorganic nanoparticles. Chemical Reviews, 104(9), 3893-3946.

[25] Derman S, Kizilbey K and Akdeste ZM. (2013). Polymeric nanoparticles. Journal of Engineering and Natural Sciences, 31, 107-120.

[26] Zhang Y, Zhai M, Chen Z, Han X, Yu F, Li Z, Xie X, Han C, Yu L, Yang Y and Mei X. (2017). Dual-modified liposome codelivery of doxorubicin and vincristine improve targeting and therapeutic efficacy of glioma. Drug Delivery, 24(1), 1045-1055.

[27] Tomii Y. (2002). Lipid formulation as a drug carrier for drug delivery. Current Pharmaceutical Design, 8(6), 467474.

[28] Kawakami S, Yamamura K, Mukai T, Nishida K, Nakamura J, Sakaeda T, Nakashima M and Sasaki H. (2001). Sustained ocular delivery of tilisolol to rabbits after topical administration or intravitreal injection of lipophilic prodrug incorporated in liposomes. Journal of Pharmacy and Pharmacology, 53(8), 1157-1161.

[29] Fetterly GJ and Straubinger RM. (2003). Pharmacokinetics of paclitaxel-containing liposomes in rats. AAPS PharmSciTech, 5(4), 1-11. 
[30] Barenholz Y. (2012). Doxil@ the first FDA approved nano-drug: lessons learned. The Journal of Controlled Release, 160(2), 117-134.

[31] Morikawa A. (2016). Comparison of properties among dendritic and hyperbranched poly(ether ether ketone)s and linear poly(ether ketone)s. Molecules, 21(2), 1-13.

[32] Chauhan AS. (2015). Dendrimer nanotechnology for enhanced formulation and controlled delivery of resveratrol. Annals of the New York Academy of Sciences, 1348(1), 134-140.

[33] Kaushik A, Tiwari S, Jayant RD, Vashist A, Nikkhah-Moshaie R, El-Hage N and Nair M. (2017). Electrochemical biosensors for early stage Zika diagnostics. Trends in Biotechnology, 35(4), 308-317.

[34] Kiraz M, Cevik S, Demirel A, Gergin YE and Ozdemir O. (2018). Nanotechnology and nanoneurosurgery. Turkish Journal of Neurochirurgie, 28(3), 264-272.

[35] Chen Y and Yang K. (2012). Intra-articular drug delivery systems for arthritis treatment. Rheumatology, 2(2), 12.

[36] Barakat NS, Bin Taleb DA and Al Salehi AS (2012). Target nanoparticles: an appealing drug delivery platform. Journal of Nanomedicine and Nanotechnology, 1-9.

[37] Romero EL. (2012). The involvement of nano-drug delivery in biosafety issues. Biosafety, 1(2), 1-5.

[38] Anwunobi AP and Emeje MO. (2012). Recent applications of natural polymers in nanodrug delivery. Journal of Nanomedicine and Nanotechnology, 4, 1-6.

[39] Torchilin VP. (2001). Structure and design of polymeric surfanctant-based drug delivery systems. The Journal of Controlled Release, 73(2-3), 137-172.

[40] Zhang X, Huang Y and Li S. (2014). Nanomicellar carriers for targeted delivery of anticancer agents. Therapeutic Delivery, 5(1), 53-68.

[41] Sayiner 0 and Comoglu T. (2016). Targeting with nanocarrier systems. Journal of Faculty of Pharmacy of Ankara University, 40(3), 62-79.

[42] Yadav HKS, Al Halabi NA and Alsalloum GA. (2017). Nanogels as novel drug delivery systems-a review. Journal of Pharmacy and Pharmaceutical Research, 1, 1-8.

[43] Sultana F, Manirujjaman, Imran-Ul-Haque Md, Arafat M and Sharmin S. (2013). An overview of nanogel drug delivery system. Journal of Applied Pharmaceutical Science, 3, 95-105.

[44] Mader K. (2006). Solid Lipid Nanoparticles as Drug Carriers. In: Torchilin VP (Eds), Nanoparticulates as drug carriers. Imperial College Press, London, UK, 187-212.

[45] Abaee A and Madadlou A. (2016). Niosome-loaded cold-set whey protein hydrogels. Food Chemistry, 196, 106113.

[46] Makeshwar KB and Wasankar SR. (2013). Niosome: a novel drug delivery system. Asian Journal of Pharmaceutical Research, 16-20.

[47] Mason TG, Wilking JN, Meleson K, Chang CB and Graves SM. (2006). Nanoemulsions: formation, structure and physical properties. Journal of Physiscs-Condensed. Matter, 18, 635-666.

[48] McClemments J, Takhistov P and Weiss J. (2006). Functional materials in food nanotechnology. Journal of Food Science, 71(9), 107-116.

[49] Sezgin Z, Yuksel N and Baykara T. (2003). Preparation and characterization of polymeric micelles as drug carrier system. Journal of Faculty of Pharmacy of Ankara University, 32(2), 125-142.

[50] Maeda H. (2001). The enhanced permeability and retention (EPR) effect in tumor vasculature: the key role of tumor-selective macromolecular drug targeting. Advances in Enzyme Regulation, 41, 189-207.

[51] Choi SH, Liu W, Misra P, Tanaka E, Zimmer JP, Ipe BI, Bawendi MG and Frangioni JV. (2007). Renal clearance of quantum dots. Nature Biotechnology, 25, 1165-1170.

[52] Venturoli D and Rippe B. (2005). Ficoll and dextran vs. globular proteins as probes for testing glomerular permselectivity: effects of molecular size, shape, charge, and deformability. American Journal of Physiology Renal Physiology, 288(4), 605-613. 
[53] Cabral H, Matsumoto Y, Mizuno K, Chen Q, Murakami M, Kimura M, Terada Y, Kano MR, Miyazono K, Uesaka M, Nishiyama $\mathrm{N}$ and Kataoka K. (2011). Accumulation of sub-100 nm polymeric micelles in poorly permeable tumours depends on size. Nature Nanotechnology, 6, 815-823.

[54] Redhead HM, Davis SS and Illum L. (2001). Drug delivery in poly (lactide-co-glycolide) nanoparticles surface modified with poloxamer 407 and poloxamine 908: in vitro characterisation and in vivo evaluation. The Journal of Controlled Release, 70, 353-363.

[55] Singh R and Lillard JR. (2009). Nanoparticle based drug delivery. Experimental and Molecular Pathology, 215223.

[56] Parveen S, Misra R and Saho SK. (2012). Nanoparticles: a boon to drug delivery, therapeutics, diagnostics and imaging. Nanomedicine, 8(2), 147-166.

[57] Danhier F, Feron 0 and Préat V. (2010). To exploit the tumor microenvironment: passive and active tumor targeting of nanocarriers for anti-cancer drug delivery. Journal of Controlled Release, 148(2), 135-146.

[58] Perez Herrero E and Fernandez Medarde A. (2015). Advanced targeted therapies in cancer: drug nanocarriers, the future of chemotherapy. European Journal of Pharmaceutics and Biopharmaceutics, 52-70.

[59] Torchilin V. (2011). Tumor delivery of macromolecular drugs based on the EPR effect. Advanced Drug Delivery Reviews, 63(3), 131-135.

[60] Bae Y and Park K. (2011). Targeted drug delivery to tumors: myths, reality and possibility. The Journal of Controlled Release, 153(3), 198-205.

[61] Baudino TA. (2015). Targeted cancer therapy: the next generation of cancer treatment. Current Drug Discovery Technologies, 12(1), 3-20.

[62] Rizvi SAA and Sale AM. (2018). Applications of nanoparticle systems in drug delivery technology. Saudi Pharmaceutical Journal, 26, 64-70.

[63] Somani S and Dufes C. (2014). Applications of dendrimers for brain delivery and cancer therapy. Nanomedicine (Lond), 9(15), 2403-2414.

[64] Quintana A, Raczka E, Piehler L, Lee I, Myc A, Majoros I, Patri AK, Thomas T, Mule J and Baker JR. (2002). Design and function of a dendrimer-based therapeutic nanodevice targeted to tumor cells through the folate receptor. Pharmaceutical Research, 19(9), 1310-1316.

[65] Dinesh B, Bianco A and Menard-Moyon C. (2016). Designing multimodal carbon nanotubes by covalent multifunctionalization. Nanoscale, 8(44), 18596-18611.

[66] Zhang Q, Yang W, Man N, Zheng F, Shen Y, Sun K, Li Y and Wen LP. (2009). Autophagy-mediated chemosensitization in cancer cells by fullerene C60 nanocrystal. Autophagy, 5(8), 1107-1117.

[67] Arafa MG, El-Kased RF and Elmazar MM. (2018). Thermoresponsive gels containing gold nanoparticles as smart antibacterial and wound healing agents. Scientific Reports, 8, 1-15.

[68] Sanad RA and Abdel-Bar HM. (2017). Chitosan-hyaluronic acid composite sponge scaffold enriched with andrographolide-loaded lipid nanoparticles for enhanced wound healing. Carbohydrate Polymers, 173, 441-450.

[69] Singh L, Kruger HG and Maguire GEM. (2017). The role of nanotechnology in the treatment of viral infecions. Therapeutic Advances in Infectious Disease, 4(4), 105-131.

[70] Chiodo F, Marradi M, Calvo J, Yuste E and Penades S.(2014). Glycosystems in nanotechnology: gold glyconanoparticles as carrier for anti-HIV prodrugs. Beilstein Journal of Organic Chemistry, 10, 1339-1346.

[71] Sakaguchi S, Yamaguchi T, Nomura T and Ono M. (2008). Regulatory T cells and immune tolerance. Cell, 133(5), 775-787.

[72] Méndez-Lagares G, Jaramillo-Ruiz D, Pion M, Pion M, Leal M, Muñoz-Fernández MA, Pacheco YM and CorreaRocha R. (2014). HIV infection deregulates the balance between regulatory T cells and IL-2-producing CD4 T cells by decreasing the expression of the IL-2 receptor in Treg. Journal of Acquired Immune Deficiency Syndromes, 65(3), 278-282.

[73] Jaramillo-Ruiz D, De La Mata FJ, Gómez R, Correa-Rocha R and Muñoz-Fernández MA. (2016). Nanotechnology as a new therapeutic approach to prevent the HIV-infection of Treg cells. PLos One, 11(1), 1-15. 
[74] Wang J, Feng SS, Wang S and Chen ZY. (2010). Evaluation of cationic nanoparticles of biodegradable copolymers as siRNA delivery system for hepatitis B treatment. International Journal of Pharmaceutics, 400(1-2), 194-200.

[75] Lebre F, Borchard G, Faneca H, Pedroso de Lima MC and Borges O. (2016). Intranasal administration of novel chitosan nanoparticle/DNA complexes induces antibody response to hepatitis B surface antigen in mice. Molecular Pharmaceutics, 13(2), 472-482.

[76] Moon JS, Lee SH, Han SH, Kim EJ, Cho H, Lee W, Kim MK, Kim TE, Park HJ, Rhee JK, Kim SJ, Cho SW, Han SH and Oh JW. (2016). Inhibition of hepatitis $C$ virus in mouse models by lipidoid nanoparticle-mediated systemic delivery of siRNA against PRK2. Nanomedicine, 12(6), 1489-1498.

[77] Sjogren MH. (2005). Prevention of hepatitis B in non-responders to initial hepatitis B virus vaccination. The American Journal of Medicine, 118, 34-39.

[78] Arya AK, Kumar L, Pokharia D and Tripathi K. (2008). Applications of nanotechnology in diabetes. Digest Journal of Nanomaterials and Biostructures, 3(4), 221-225.

[79] Cui F, Qian F, Zhao Z, Yin L, Tang C and Yin C. (2009). Preparation, characterization, and oral delivery of insulin loaded carboxylated chitosan grafted poly (methyl methacrylate) nanoparticles. Biomacromolecules, 10(5), 1253-1258.

[80] Harsoliya MS, Patel VM, Modasiya M, Pathan JK, Chauhan A, Parihar M and Ali M. (2012). Recent advances and applications of nanotechnology in diabetes. International Journal of Pharmaceutical and Biological Archive, 3, $255-261$.

[81] Meetoo D and Lappin M. (2009). Nanotechnology and the future of diabetes management. Journal of Diabetes Nursing, 13(8), 288-297.

[82] Gordon N. (2003). Sagman, U. Nanomedicine taxonomy. Briefing paper.

[83] Hanazaki K, Nose Y and Brunicardi FC. (2001). Artificial endocrine pancreas. Journal of the American College of Surgeons, 193(3), 310-322.

[84] Freitas RA. (2005). Current status of nanomedicine and medical nanorobotics. Journal of Computational and Theoretical Nanoscience, 2, 1-25.

[85] Chanda AV and Unnikrishnan V. (2018). Novel insole design for diabetic foot ulcer management. Proceedings of the Institution of Mechanical Engineers, Part H, 232(12), 1182-1195.

[86] Sahoo SK, Parveen S and Panda JJ. (2007). The present and future of nanotechnology in human health care. Nanomedicine, 3(1), 20-31.

[87] Naschie MSE. (2006). Nanotecnology for the devoloping world. Chaos, Solution and Fractals, 30(4), 769-773.

[88] Lafisco M, Ruffini A, Adamiano A, Sprio S and Tampieri A. (2014). Biomimetic magnesium-carbonate-apatite nanocrystals endowed with strontium ions as anti-osteoporotic trigger. Materials Science and Engineering: $\mathrm{C}, 35$, 212-219.

[89] Balasundaram G and Webster TJ. (2010). HA coated magnetic nanoparticles for the treatment of osteoporosis. $27(9)$.

[90] Huang S, Chen JC, Hsu CW and Chang WH. (2009). Effects of nano calcium carbonate and nano calcium citrate on toxicity in ICR mice and on bone mineral density in an ovariectomized mice model. Nanotechnology, 20(37), 1-7.

[91] Tokudome Y, Ito A and Otsuka M. (2011). Effect of zinc-containing $\beta$-tricalcium phosphate nano particles injection on jawbone mineral density and mechanical strength of osteoporosis model rats. Biological and Pharmaceutical Bulletin, 34(8), 1215-1218.

\section{How to cite this article}

Yerebasan U and Gul Baykalir B. (2020). The importance of nanotechnology and drug carrier systems in pharmacology. GSC Biological and Pharmaceutical Sciences, 10(2), 14-23. 